\title{
An array-based study of increased system lifetime probability
}

\author{
Nesgaard, Carsten
}

Published in:

APEC 2003: Eighteenth Annual IEEE Applied Power Electronics Conference and Exposition

Publication date:

2003

Document Version

Publisher's PDF, also known as Version of record

Link back to DTU Orbit

Citation (APA):

Nesgaard, C. (2003). An array-based study of increased system lifetime probability. In APEC 2003: Eighteenth Annual IEEE Applied Power Electronics Conference and Exposition (Vol. 1-2, pp. 476-482). IEEE. I E E E Applied Power Electronics Conference and Exposition. Conference Proceedings

\section{General rights}

Copyright and moral rights for the publications made accessible in the public portal are retained by the authors and/or other copyright owners and it is a condition of accessing publications that users recognise and abide by the legal requirements associated with these rights.

- Users may download and print one copy of any publication from the public portal for the purpose of private study or research.

- You may not further distribute the material or use it for any profit-making activity or commercial gain

- You may freely distribute the URL identifying the publication in the public portal

If you believe that this document breaches copyright please contact us providing details, and we will remove access to the work immediately and investigate your claim. 


\title{
An array-based study of increased system lifetime probability
}

\author{
Carsten Nesgaard, Member IEEE \\ Department of Electrical Power Engineering \\ Technical University of Denmark \\ 2800 Kongens Lyngby \\ Email: carsten@nesgaard.com
}

\begin{abstract}
Society's increased dependence on electronic systems calls for highly reliable power supplies comprised of multiple converters working in parallel. This paper describes a redundancy control scheme, based on the array technology that increases the overall reliability quite considerably and thereby ensures a stable supply voltage.
\end{abstract}

\section{INTRODUCTION}

With the ever-increasing dependence on reliable electronic systems, the area of highly reliable power systems is rapidly expanding. When considering highly reliable fault tolerant power systems the word 'redundancy' comes to mind. Indeed, a true fault tolerant power system is comprised of several converters working in parallel. This paper describes part of an ongoing project of building a prototype of a fault tolerant power system with $\mathrm{N}+2$ redundancy. The paper gives a short description of the mathematics used, the power system in question and the choice of control scheme. Since each of these short descriptions can form the basis for an entire paper, the focal point in this presentation is the redundancy control within the overall power system.

\section{ARRAY-BASED LOGIC}

Originated at the Department of Electric Power Engineering, the Technical University of Denmark in 1978 with the paper "Group Representations of Finite Polyvalent Logic - a Case Study Using APL Notation" by associate professor Ole I. Franksen [4], the array-based logic has evolved into an effective tool when dealing with combinatorial and/or configuration applications. The foundation of the technology is a geometrical representation of logic in terms of nested arrays. In other words, the array-based concept deals with data objects regarded as arrays [7]. Consequently, all calculations are performed on arrays which implies that systems comprised of large amounts of data often can be systematically simplified by the use of array theoretical operations. In general, the arraybased logic can be considered to consist of the following three steps:
Step one is the establishment of a discrete n-dimensional configuration space using the Cartesian product, which ensures completeness. This is accomplished by the use of the tensor product OUTER and [8], which combines the system propositions and unites them to form one conjunctive proposition.

Step two is the inference by colligation, which is the operation of establishing the interconnections of the system. In other words this step finds the solutions that comply with the system constraints.

Step three is the determination of states by elimination of variables through an or-reduction.

Having introduced the concept of constraints, it is obvious that prior to completion of the above-mentioned steps, the constraints of the physical system must be translated into array theoretical terms. This is achieved through the use of propositional logic that transforms the system constraints into logic operations suitable for array theoretical implementation. An example of this translation and implementation is shown in section 'V. ARRAY-BASED CONTROL SCHEME'.

Summarizing the above description the basic idea of arraybased logic can be expressed, according to Franksen, as:

Array-based logic explores the consequences of considering truth-values as physical measurements. The aim is to formalize logic in accordance with the theoretical structure of discrete systems and express this formalization algebraically in arraytheoretic terms.

\section{REDUNDANCY AS RELIABILITY ENHANCEMENT}

Increasing the reliability of a power system can be achieved in a number of ways, some more successful than others. A simple way of increasing the overall system reliability is using high quality components with low failure rates. Although, the overall circuit complexity remains the same the cost for high quality components is considerably higher than that of an implementation using commercial components. Also, the reliability gain using these high quality components is only moderate compared to other reliability enhancement techniques. 
For the reasons mentioned above, by far the most common way of increasing the reliability is the use of redundancy. Once the choice of redundancy has been made the level of redundancy needed must be identified. Ranging from the component level all the way to the system level, the level of redundancy used in the power system in this presentation is what can be characterized as block level redundancy.

As with any system, a redundant power system has both advantages and drawbacks. Among the advantages is the possibility of a dramatic increase in reliability at the expense of an increase in system dependent parameters such as cost, mass, volume and circuit complexity. Although the increase in reliability can be quite high, added cost, mass, volume, and complexity are drawbacks that must be considered when deciding which approach to take during the design phase of the power system. However, the drawbacks tend to be less important nowadays, since system downtime in case of power failure often results in greater losses in sales, customer services etc.

When implementing system level redundancy in power systems, each converter board within the overall power system must be equipped with a front switch that allows for controlled shutdown of faulty converter boards, since this is the only way the power system integrity can be maintained. In other words the power system must exhibit failure free operation at the input as well as at the output. Due to this fact, most approaches in designing high reliable power systems originates from the ability of the overall power system to shutdown faulty units whether these consists of converter boards, power distribution units etc. Focusing on the reliability of redundant systems it is noteworthy that making a single path system redundant generally increases the overall reliability with a factor of the reciprocal of the initial failure rate for the single path system, assuming the exponential distribution is valid and that the working conditions in both cases remain unchanged.

Now, suppose the redundant power system had the ability to reconfigure itself during operation. Such a system would further increase the reliability of the overall power system and at the same time reduce the maintenance requirements, since faulty units could be 'replaced' automatically. Due to the dynamic process of continuous measurement of the system integrity and configuration at any given time the simple exponential distribution for reliability calculations does not justify the true reliability potential in a reconfigurable system. To obtain a more truthful measure for the system reliability, one has to adopt the use of Markov modeling techniques, which is a commonly used modeling technique dealing with dynamic systems. Although Markov modeling often includes random noise errors within the sensor feedback system, it is for simplicity assumed that such errors do not occur within the power system at hand. The justification for making this simplification is that the focal point in this presentation is the redundancy control scheme. Furthermore, since the sensor feedback system is discrete with logic truth-values, the noise margin is quite large and random noise errors would be extremely rare in any case. The detailed description of Markov modeling uses can be found in the literature [6] and will not be dealt with in this presentation.

\section{THE POWER SYSTEM}

It has been chosen to investigate a power system comprised of 5 identical converter boards connected in parallel. As indicated in the introduction this is 2 converter boards more than needed. Thus, the system is $\mathrm{N}+2$ redundant. On a board level each converter is designed to shutdown in case of a single point failure whereas the overall power system can tolerate 2 failures and still provide the needed power. From a traditional power system point of view two failures reduces the overall power system from a system comprised of 5 parallel-connected converter boards into a system comprised of the 3 converter boards needed to supply the required power to the load.

The proposed power system approaches the parallel connection of the individual converter boards in a way that differs significantly from what have just been described, by splitting the individual converter boards into 5 main blocks. Aligning these main blocks as shown in Figure 1 (ignoring the block 'PWM controller'), each block connects to the previous block on the same converter boards as well as to the previous blocks on the parallel-connected converter boards. This arrangement of multiple inter-connection of individual blocks allows for intelligently control of combining blocks for maximum number of working converter boards at any given time. It should be noted that it is not an allowable state to have a block deliver power to more that one subsequent block. Thus, the first system constraint is the limitation of blocks being connected to one and only one subsequent block.

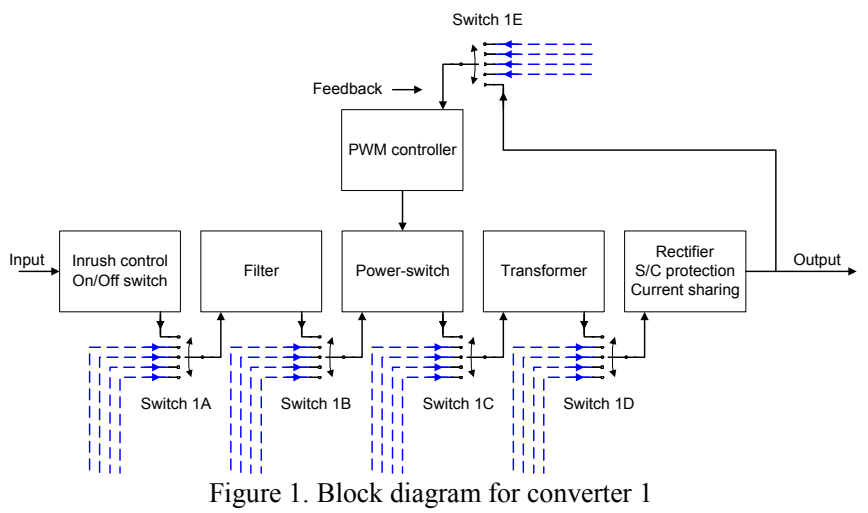

The connecting devices are chosen to be electronically controlled switches but could in theory also be mechanically operated relays. The reason for choosing electronically controlled switches (hereafter referred to as switches) is the fact that activation of the individual interconnecting devices would occur during power system operation, which for long switching periods would require substantial amounts of capacitors at the output of the power system in order to comply with ripple voltage specification. Thus, the timing of the switching is of importance although not critical. A transition 
time of $0.1 \mathrm{~ms}$ is estimated to be reasonable. Compared to the $140 \mathrm{kHz}$, which has been chosen as the switching frequency for the individual converter boards, it is apparent that the transition times of the interconnecting switches are far from critical.

Even though the interconnecting switches are operated rarely, due to the rather low failure rate of the electronic components used and the transition time from one state to another is fairly quick, the price paid for using extra switches as connecting devices between the different blocks within the overall power system is an increased failure rate for the individual converter boards and an increase in total conduction losses. Furthermore, the overall cost and complexity of the power system is increased due to the use of extra switches. However, if these drawbacks at the board level imply a better probability of continuous operation at a system level, the added cost, complexity and losses might be negligible compared to the gain in reliability. Also, it should be noted that obtaining a similar reliability for a power system comprised of individual converter boards without the interconnecting capability would require more converter boards, which adds to system parameters such as volume and mass.

As mentioned in section 'III. REDUNDANCY AS RELIABILITY ENHANCEMENT' the statistical description of the reliability of the power system should be carried out using the Markov modeling approach. However, from a system point of view it should be obvious that the process of combining two defective converter boards to form one working converter board increases the overall system performance concerning both reliability and efficiency. In Figure 2 it can be seen that by combining two converter boards, which have failed in different locations on the board level, an alternative path for the power throughput can be established. This lowers the stress on the original 3 converter boards, since the load current now is shared among 4 converter boards. As a consequence the power system operating point on the efficiency curve tends to move towards the optimum operating point as shown in Figure 3. Also and most importantly, the overall power system reliability increases as a result of the newly configured power system comprised of $3+1$ working converter boards.

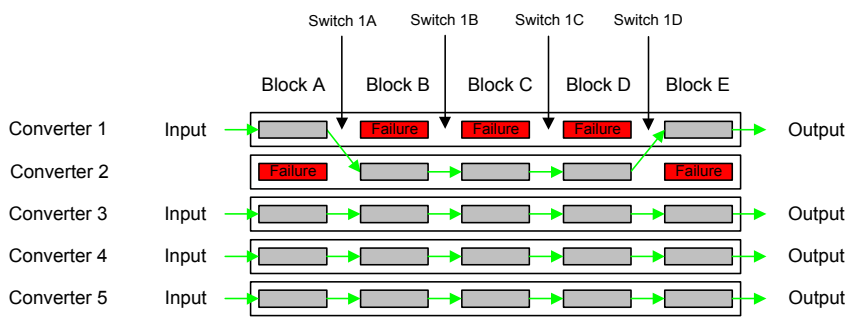

Figure 2. The 5 parallel connected converter boards

Referring to Figure 1 and Figure 2 this paragraph describes the abbreviations used to identify the individual blocks and switches within the power system. Starting with the blocks it can be seen from Figure 2 that these can be addressed using the converter number as a row identification and the block letter as column identification. Thus, the first faulty location in the power system shown in Figure 2 can be identified as: Converter 1, Block B.

Identification of the interconnection switches is accomplished through the adoption of the following notation: SXYZ

Where $\mathrm{S}$ is the notation used in the software to identify a switch. X represents the converter number, $\mathrm{Y}$ represents the block prior to the switch in question and $\mathrm{Z}$ represents the switch position. Hence, the switch between block $1 \mathrm{~A}$ and block $1 \mathrm{~B}$ set in position 1 gets the identification S1A1.

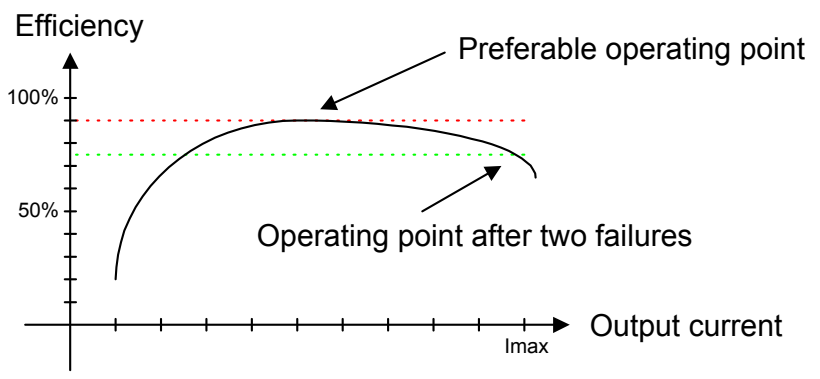

Figure 3. Example of operating point movement

In order to provide feedback to the redundancy control system, each block in the overall power system can take on two different logic values - logic 1 for a working block and logic 0 for a faulty block. Since a faulty block is switched off and the redundancy control system continues to check the status of the overall power system, the logic state of any faulty block is latched. This ensures that the redundancy control system always gets the correct logic values from each block, even though the block in question has failed. Having retrieved all truth-values from the blocks within the power system an array containing the retrieved truth-values is generated. As will become apparent in section ' $\mathrm{V}$. ARRAY-BASED CONTROL SCHEME' this array forms the basis for the calculation process as well as for the representation of the results.

Turning the attention towards the operation of the power system the following description represents the actions taken by the redundancy control in case of fault occurrence. Assuming a well functioning structure as the starting point, the power system consists of 5 inputs and 5 outputs. After a failure within the power system, the redundancy control shuts down the blocks associated with the faulty block and leaves the power system comprised of 4 inputs and 4 outputs. Except for the faulty block the rest of the inactive blocks now serve as cold spares for the power system in case of further failures. Now suppose a second fault occurs, for instance due to the increased stress on the remaining active converter boards. Since two faults have occurred it might now be possible to establish an alternative path through the power system and thus increase the number of active converter boards from 3 to 4 . The only constraint that makes the establishment of an alternative path impossible is in case the two faults have occurred within the same block. In this case the power system would consist of 
only 3 converter boards, which is the minimum number possible in order to sustain power delivery to the load.

Depending on the failure rate of the individual blocks it would be a rare situation that two successive faults occur in the same block, hence the probability of successful system reconfiguration is quite high. This indicates that the overall system reliability has increased compared to the situation with 5 separate converter boards.

Based on this short description of the power system and its operation, the mathematical task of the redundancy control can be thought of as a method of finding alternative paths through the power system in case of fault occurrence.

\section{ARRAY-BASED CONTROL SCHEME}

As described in section 'II. ARRAY-BASED LOGIC' the mathematical foundation is a consideration of truth-values as physical measurements. The truth-values in the application at hand are the discrete values obtained from each block in the power system, upon which the alternative path from input to output is calculated. With reference to Figure 2 it can be seen that the discrete values obtained from the individual blocks can be considered as an array of 5 rows and 5 columns. This array is a measurement of the condition of the overall power system and can therefore be used to identify problems within the system. Based on this identification of problems, the arraybased analysis suggests possible alternative paths through the power system. It should be noted that similar results could have been obtained by using standard digital logic. The reason for not implementing the redundancy control using this type of logic is due to the powerful array concept and operations in array theory, which makes it easy to expand the redundancy control scheme to include an arbitrary number of converter boards and switches. Thus, a formal description of a redundant power system comprised of any number of parallel-connected converter boards is straight forward, since the added system constraints are almost replicates of existing board level constraints. Similar implementation using standard digital logic would require considerable recalculations of the power system's interconnections.

With reference to Figure 1 and Figure 2 it can be seen that the number of parameters needed to describe the power system in question is relatively large. For this reason most system constraints have been omitted in this presentation, although an example will be given on the following pages.

Having introduced the fundamentals of the power system, the array-based analysis can be carried out. The mathematical tool used in this project is based on the array theory developed by Dr. Trenchard More in the 1970's and later (early 1980's) implemented in the array-based software 'Queens Nested Interactive Language (Q'Nial)' by Professor Michael Jenkins.

Solving the problem at hand by establishing a generalized configuration space by the use of the Cartesian product of all system parameters would require a tremendous amount of computer memory, since the number of possible combinations exceeds $10^{30}$. A different approach has therefore been pursued.
Using the allowable positions for each switch, an algorithm has been developed that in a successive way finds a feasible solution within a given amount of time.

Looking at the power system and the tasks of the redundancy control from a topological point of view it should be noted that the system's topological array has characteristics similar to that of the incidence matrix describing electric networks within the field of graph theory. The reason the topological array only has similar and not identical characteristics to the incidence matrix is due to the unidirectional flow of power through the system. The classical approach in electric network theory using arrays is the bi-directional power flow that uses the numbers 0,1 and -1 to identify the flow direction. Since the power system at hand only allows power to flow in one direction the closest match to the incidence matrix is the use of unidirectional circuit elements such as semiconductor devices within the electric network itself. From a mathematical point of view this adds considerably to the complexity of the system when performing reliability calculations, since the system now includes multiple failure modes for each block. Also, it should be noted that the analysis assumes a constraint between the blocks 'PWM controller' and 'Power-switch'. This constraint ensures a correct connection between the driving 'PWM controller' and the semiconductor device making up the switching element of the converter.

As mentioned above the starting point in the development of the software was a series of system constraints that would limit the number of allowable switch combinations. The implementation example used in this presentation is the constraint saying that a switch set in position 1 cannot be set in any other position. Describing this constraint with a 'logiclevel' explanation, one obtains:

\section{If $\mathrm{s} 1 \mathrm{a} 1=1$ Then $\mathrm{s} 1 \mathrm{a} 2 \& \mathrm{~s} 1 \mathrm{a} 3 \& \mathrm{~s} 1 \mathrm{a} 4 \& \mathrm{~s} 1 \mathrm{a} 5=0$}

This description must now be converted into Nial terms, which results in the following lines of source code:

a2:= OUTER $<=$ s1a1 (not s1a2);

a3:= OUTER $<=$ s1a1 (not s1a3);

a4:= OUTER $<=$ sla1 (not sla4);

a5:= OUTER $<=$ s1a1 (not s1a5);

Noting the replication of parameter s1a1, the proposition must be united through the operation of colligation:

\section{$(024$ 6) (1) (3) (5) (7) fuse (OUTER and a2 a3 a4 a5)}

Following the above procedure, the remaining system constraints can be added to the source code. After a few transformations a list of allowable switch positions based on the system constraints can be obtained. Keeping in mind that the initial $2^{5}$ combinatorial switch positions for any given switch now has been reduced to 6 allowable switch positions that comply with the system constraints: 


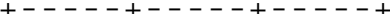

|00000|00001|00010|00100|01000|10000|

The first entry from the left in (1) is the NULL solution where the block subsequent to the switch in question is disconnected from all blocks within the power system. The entry to the right in (1) is the notation used for switch position 1 . The second entry from the right is the notation used for switch position 2 and so forth. As an example the entry to the right in (1) indicates that the left-hand side of the switch in question is connected to Converter 1 regardless to which converter board the right-hand side of switch is connected. Thus, a ' 0 ' in any entry in (1) indicates a disconnection whereas a ' 1 ' indicates a connection between two blocks.

Since the software program decides which blocks to interconnect at all times the power system would under normal circumstances interconnect the individual block in a way that enables power throughput within the physical boundaries of each converter board as shown in Figure 2. In case of multiple failures the algorithm would find a way through the system that ensures a maximum number of working converter boards. In other words, allowing the algorithm to decide which blocks to interconnect, the overall power system is no longer comprised of 5 individual converter boards with interconnecting switches, but 5 times 5 blocks that can be interconnected in an enormous number ways. This gives rise to the previously mentioned increase in reliability. As a consequence, a high reliable power system can be built with lower volume and mass than conventional power systems, but at the cost of increased circuit complexity and considerably higher cost price.

As an example of the capabilities of the algorithm, let the power system suffer from 8 faults located in different places throughout the power system. A condition from which a power system comprised of standard parallel-connected converter boards could not recover. As seen from Figure 4 each row in the power system has suffered at least one failure.
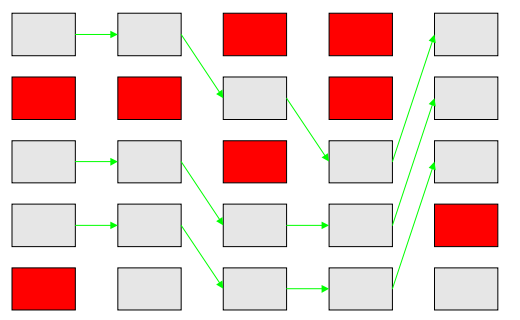

\begin{tabular}{|l|l|l|l|l|}
\hline 1 & 1 & 0 & 0 & 1 \\
\hline 0 & 0 & 1 & 0 & 1 \\
\hline 1 & 1 & 0 & 1 & 1 \\
\hline 1 & 1 & 1 & 1 & 0 \\
\hline 0 & 1 & 1 & 1 & 1 \\
\hline
\end{tabular}

Figure 4. 8 faults distributed among all 5 converters

The array to the right in Figure 4 is the system truth-values as they are entered into the system array for calculation purposes. The software program now performs the following tasks in order to establish a path between the first two blocks. The starting point is the answer to the following question:

Does the combination of 11 occur in the first two columns in the matrix shown to the right in Figure 4? In Nial terms this can be expressed in a very compact form:

\section{$\mathrm{Q}:=((0$ pick $(\operatorname{cols}$ AA $)$ EACHLEFT $=11)$ link o $)$}

lolloo

The result is shown as truth-values. It should be noted that due to the number of allowable switch positions a falsehood has been attached to the end of the result.

Assigning the correct switch positions to the entries that returned truth is completed through the operation 'sublist'.

$\mathrm{Y}:=\mathrm{Q}$ sublist (reverse Res_1)

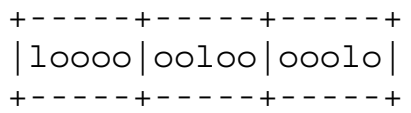

In order to insert the correct switch positions into the result array, the positions that returned truth must be identified in Index origin 0.

\section{Index:= EACH first (Y EACHLEFT sublist tell (first shape AA))}

\section{3}

Finally, the assigned switch positions are inserted into the result array by using the operation 'placeall'.

\section{Y (cart Index 0) placeall AA}

Following a similar procedure the rest of the truth-values in the array shown to the right in Figure 4 are replaced by feasible switch positions. The resulting array for the case of 8 faults distributed among all 5 converters is shown in Figure 5.

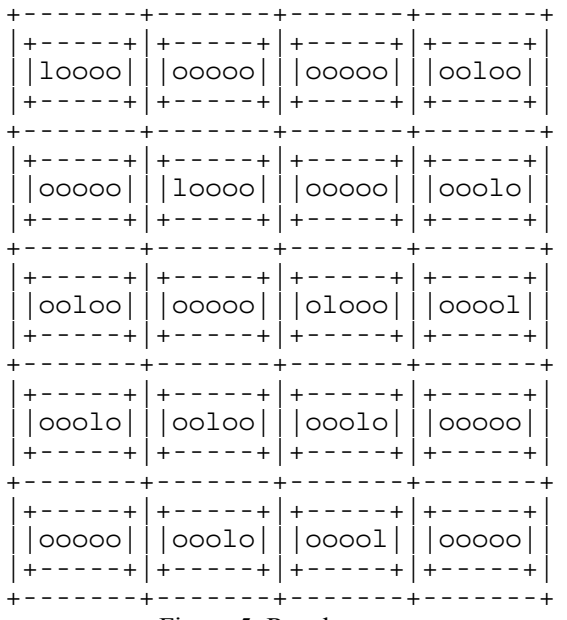

Figure 5. Result array

Comparing the array shown to the right in Figure 4 with the result array shown in Figure 5 it is obvious that the two arrays 
are linked through a transformation array. Considering the axes of the two arrays it can be seen that the transformation array is the previously mentioned incidence matrix for electric networks.

\section{RELIABILITY ASSESSMENT}

As indicated in section 'III. REDUNDANCY AS RELIABILITY ENHANCEMENT' the reliability of the power system at hand is best found using the Markov Modeling approach. However, due to the large number of states in which the power system can reside the calculations become extremely complicated resulting in loss of any insight into the relation between survivability of each block and the impact of the overall system. Changing the viewpoint from dynamic parts level redundancy to system level survivability makes it possible to express the overall system performance concerning reliability as a function of time. It should be noted that this approach does not provide any system information during transition from one state to another. However, in most cases the figure of merit relevant to most customers is the probability of system survival within the expected system lifetime. For this reason the proposed system level approach will be utilized.

When considering reliability assessment of systems several evaluation techniques are applicable. However, due to the complicated interconnection of the individual blocks within the power system a generalized approach focusing on a formal system description by means of block reliabilities is desirable. Two approaches comply with the latter desire - event trees and connection matrix techniques. Since the power system at hand is comprised of a rather large number of blocks the event tree approach quickly becomes too complex. In contrast the connection matrix technique establishes a matrix representing power flow between system nodes by means block reliabilities. Thus, the obvious approach is the connection matrix technique, which will be used throughout the remainder of this presentation.

Figure 6 shows a cross section of the power system found in Figure 2.

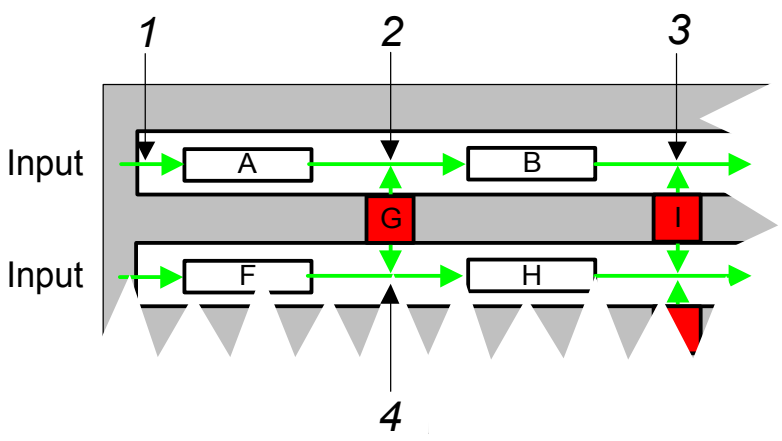

Figure 6. Cross section of Figure 2

Representing both power flow and system nodes the green arrows (horizontal) in Figure 6 are the basis for the connection matrix technique. It should be noted that flow from one block to another is unidirectional whereas the flow to and from a switch is bidirectional. The blocks interconnecting the individual nodes are characterized by their probability of providing fault free operation for a specified period of time. The establishment of the connection matrix is now straightforward as the entries of the matrix are the probabilities for each block interconnecting two adjacent nodes. Figure 7 shows the technique applied to the blocks and nodes found in Figure 6.

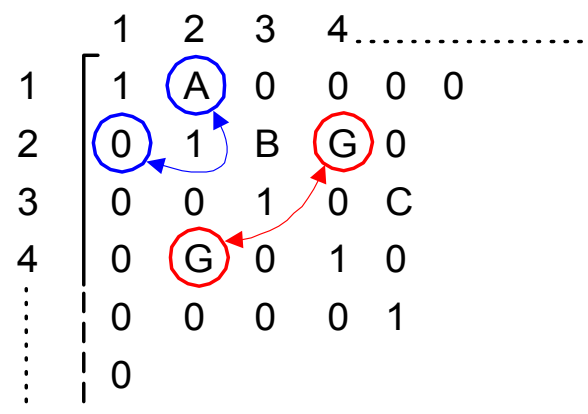

Figure 7. Connection matrix for

In Figure 7 the blue circles define the unidirectional flow between node 1 and 2 (block A) while the red circles define the bidirectional flow between node 2 and 4 (block G - a switch).

Having established the entire connection matrix the next step is either node removal through sequential reduction or matrix multiplication. The latter method being the easiest to apply, thus being the method preferred. Application of the matrix multiplication is straightforward as the basic connection matrix is multiplied by itself a number of times until the resulting matrix remains unchanged. The transmission from input to output is now derived from the matrix as the entry found in row ' 1 ' and the column containing the output node.

Based on the solution derived from the connection matrix and on the assumption that the block failure rates throughout the power system are identical the following system probability equation can be established:

$$
\mathrm{P}_{\text {Array-based }}=\mathrm{e}^{-\left(\frac{\lambda}{5}+\lambda_{\text {Switch }}\right) \cdot \mathrm{t}}
$$

$\lambda_{\text {Switch }}$ is the failure rate of each switch and $\lambda$ is the overall failure rate for each converter board. By means of the exponential distribution the probability of system survival of a traditional redundant power system can be found as:

$$
\mathrm{P}_{\text {Traditional }}=\mathrm{e}^{-\lambda \cdot \mathrm{t}}
$$

Comparing (3) and (4) it can be seen that the difference is the exponent. As will become apparent this difference is of great importance when considering redundant systems. By means of the probabilities found in (3) and (4) the binomial coefficients for a $\mathrm{N}+2$ redundant system can be established: 


$$
\begin{aligned}
& \mathrm{R}_{\text {Traditional }}=10 \cdot \mathrm{e}^{-3 \cdot \mathrm{t} \cdot \lambda}-15 \cdot \mathrm{e}^{-4 \cdot \mathrm{t} \cdot \lambda}+6 \cdot \mathrm{e}^{-5 \cdot \mathrm{t} \cdot \lambda} \\
& \mathrm{R}_{\text {Array-based }}=10 \cdot \mathrm{e}^{-3 \cdot \mathrm{t} \cdot \kappa}-15 \cdot \mathrm{e}^{-4 \cdot \mathrm{t} \cdot \kappa}+6 \cdot \mathrm{e}^{-5 \cdot \mathrm{t} \cdot \kappa}
\end{aligned}
$$

where $\kappa$ is equal to:

$$
\kappa=\left(\frac{\lambda}{5}+\lambda_{\text {Switch }}\right) \cdot \mathrm{t}
$$

Plotting the two equations reveals the probability of system survival for a given period of time as a function of overall converter board failure rate.

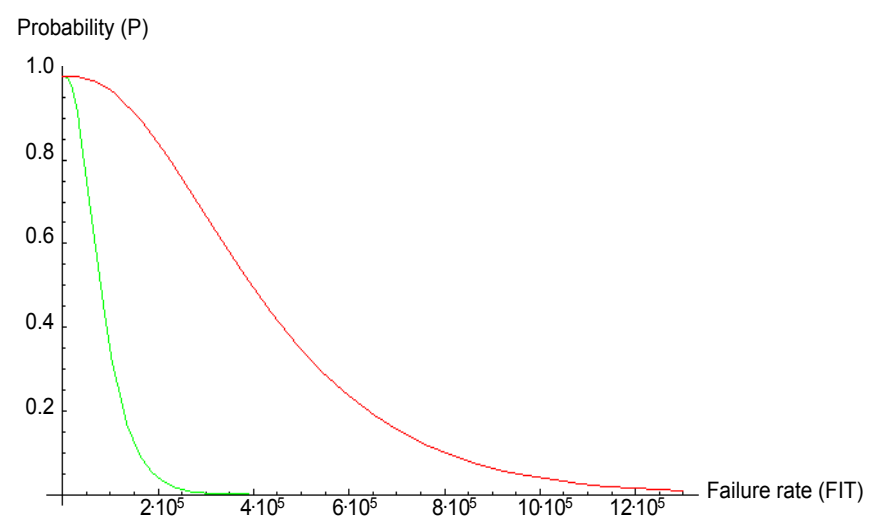

Figure 8. Probability of system survival

The red line in Figure 8 is the probability of system survival for the array-based approach while the green line shows the probability of system survival for a traditional redundant power system.

The reliability of the array-based approach is worse at converter board failure rates below the switch failure rate plus one-fifth the converter board failure rate. The boundary between the two reliability scenarios can in mathematical terms be expressed as:

$$
-\left(\frac{\lambda}{5}+\lambda_{\text {Switch }}\right) \cdot \mathrm{t}=-\lambda \cdot \mathrm{t} \Rightarrow \lambda=\frac{5 \cdot \lambda_{\text {Switch }}}{4}
$$

Thus, at converter board failure rates below the value given in (8) the traditional approach would be preferable. However, the converter board failure rate for any power system would by far exceed the failure rate of a single switch. For this reason it can be concluded that the array-based approach indeed increases the overall reliability of the proposed power system configuration.

\section{CONCLUDING REMARKS}

An alternative approach in the design of reliable power systems has been presented. Based on statistical calculations using among others the exponential distribution it has been show that redundancy is the tool to implement when considering high reliable power systems.

Also, a control scheme for the redundancy control of the power system has been presented. Using the array-based logic a well functioning system capable of establishing the maximum number of working converter boards possible has been implemented.

Finally, an assessment of the overall gain in power system reliability has been performed. This assessment showed that a considerable increase in system survivability is possible when the proposed array-based control and implementation technique is applied.

\section{ACKNOWLEDGMENT}

The author would like to thank Associate Professor, Ph.D. Peter Falster, Department of Electric Power Engineering, Technical University of Denmark and Senior Designer Henrik Møller, Alcatel Space Denmark for their support during this work.

\section{REFERENCES}

[1] Military Handbook (MIL-HDBK-217): Reliability Prediction of Electronic Equipment.

[2] Mike Jenkins and Peter Falster: “Array Theory and Nial”, report 1999, Department of Electric Power Engineering, Technical University of Denmark.

[3] Gert L. Møller: "On the technology of array-based logic", Ph.D. thesis 1995, Department of Electric Power Engineering, Technical University of Denmark.

[4] Ole Immanuel Franksen: "Group Representations of Finite Polyvalent Logic - a Case Study Using APL Notation", IFAC VII World Congress, Helsinki, June 1978.

[5] Ole Immanuel Franksen and Peter Falster: "Colligation or, the logic inference of interconnection", Mathematics and Computers in Simulation $52(2000) 1-9$.

[6] Bruce K. Walker: "Evaluating Performance and Reliability of Automatically Reconfigurable Aerospace Systems Using Markov Modeling Techniques", Department of Aerospace Engineering \& Engineering Mechanics, University of Cincinnati, OH, USA.

[7] Trenchard More: "Notes on the Diagrams, Logic and Operations of Array Theory", Structures and operations in Engineering and Management Systems, The second Lerchendal Book, Tapir Publishers.

[8] Mike A. Jenkins: “Q'Nial Reference Manual”, Nial Systems Limited, Kingston, Ontario, Canada, 1985. http://www.nial.com 